\title{
Comparison of extraction efficiency of three nanofibers for four heavy metals in aqueous solution
}

Xiongwei Liu1, a , Jianjun Deng ${ }^{2, \text { b }}$, Jinghui Fan ${ }^{1, c}$, Lanlan Wei, ${ }^{3, d}$, Xuejun Kang ${ }^{1 *, e}$

${ }^{1}$ Key Laboratory of Child Development and Learning Science (Ministry of Education), School of Biological Science and Medical Engineering, Southeast University, Nanjing 210096, China

2 Suzhou Dongqi Biological Technology Co., LTD, Suzhou 215123, China

${ }^{3}$ Key Laboratory of Environmental Medicine and Engineering, Ministry of Education,

School of Public Health, Southeast University, Nanjing 210009, China

aliuxiongwei0630@163.com, exjkang64@163.com

\section{Keywords: Nanofibers; heavy metals; ICP-MS; trace concentration}

Abstract. A new conductive polymer nanofiber was prepared by electrospinning of the polymer solution and in situ synthesis. The electrospun polystyrene (PS) fibers were employed as template for the in situ surface polymerization of poly 3, 4-ethylenedioxythiophene (PEDOT). Electrospun nanofibers were utilized as a sorbent in packed fiber solid phase extraction (PFSPE) for selective separation and concentration of $\mathrm{As}^{3+}, \mathrm{Cd}^{2+}, \mathrm{Hg}^{2+}$ and $\mathrm{Pb}^{2+}$. A SPE coupled with ICP-MS was established for analysis of trace heavy metals in aqueous solution. The extractive recoveries of four metal ions on three nanomaterials, PS, PEDOT, and polystyrene - dithizone (PS-DZ) nanofibers were compared. PEDOT nanofibers appears better enrichment ability than other two nanofibers, and the average recovery of four metals is $96.3 \%$. It seems that PEDOT was especially suitable for the extraction of heavy metal ions with low concentration in water, which is of great significance in the monitor of trace metals in water sample.

\section{Introduction}

Heavy metal pollution has been highly concerned, and the removal of heavy metal pollution is particularly important. In recent years, many kinds of adsorption materials have been developed mostly for remove of heavy metal ions from sewage and industrial wastewater or for the recovery of noble metal ions in industrial wastewater. However, there are few reports on the simltaneous extraction and concentration of multiple heavy metals before analysis and monitoring of them in water at trace level [1].

Organic conducting polymer is a research field developed in 70s twentieth Century [2]. It is a kind of conjugated polymer with large $\pi$ bond. The structure is usually divided into a polymer chain and a doped pair of anions or cations. At present, because of its unique nature and wide application prospects, it has become one of the most active fields of research. Polythiophene is an important conductive polymer, characterized by rigidity, insoluble and unmelted properties, and the thermal stability of it in air is satisfactory. Poly 3, 4-ethylenedioxythiophene (PEDOT) is the polymer of 3, 4-ethoxylene dioxy thiophene (EDOT), which is the derivative of polythiophene. PEDOT is promising for uses in the field of environment because of its better environmental stability and facile synthesis. The preparation of nanofiber materials by electrostatic spinning is a valid technology to produce a polymer with nanoscale fibrous structures. Owing to their large surface area to volume ratio, electrospun nanofibers facilitate the miniaturization of solid phase extraction (SPE) when they are packed into a cartridge as the sorbent beds [3-4]. Because the direct determination of heavy metal ions at trace level in samples is limited due to their low concentrations and matrix interferences [5-6]. Our group had developed an packed fiber solid phase extraction (PFSPE) for selective separation and preconcentration of lead (II) by use of an electrospun nanofiber as a sorbent with a polystyrene (PS) backbone functionalized with dithizone (DZ) by co-electrospinning of a polystyrene solution containing dithizone (DZ)[7-8]. 
In this work, PEDOT nanofibers were prepared by using PS fiber as a template for the in situ surface polymerization of poly 3, 4-ethylenedioxythiophene. The extractive recoveries of four metal ions $\left(\mathrm{As}^{3+}, \mathrm{Cd}^{2+}, \mathrm{Hg}^{2+}\right.$ and $\left.\mathrm{Pb}^{2+}\right)$ on three nanomaterials, PS, PEDOT, and polystyrene - dithizone (PS-DZ) nanofibers were compared.

\section{Experimental}

\section{Reagents and Apparatus}

All chemicals used throughout the work were of analytical reagent grade and were used without further purification. Doubly distilled deionized water was used throughout all experiments. Polystyrene (PS) and 3, 4-ethylenedioxythiophene (EDOT) was obtained from Shanghai Chemical Agents Institute. Sodium hydroxide and nitric acid were obtained from Guangdong Chemical Agents Institute. The standard labware and glassware used were cleaned with nitric acid and rinsed with double distilled water. $\mathrm{Cd}$ ( II ), $\mathrm{Hg}$ ( II ), $\mathrm{Pb}$ ( II ), As( III) standard reserve liquid are provided by the National Center for analysis and testing.

An ICP-MS7700 (Agilent Technologies ) was used to achieve fast analysis of metal ions. A high-voltage power supply (model DW-P403-1AC, Tianjin, China), and a syringe pump were used for the electro-spinning. The nanofiber was examined using S23000N field-emission scanning electron microscope (FE-SEM, Hitachi, Japan).

\section{Preparation of PS and PEDOT nanofibers}

$10 \%(\mathrm{w} / \mathrm{v})$ PS was dissolved in a mixture of dimethylformamide and tetrahydrofuran $(4: 6, \mathrm{v} / \mathrm{v})$. Then the solutions are stirred at room temperature for $10 \mathrm{~h}$ prior to electro-spinning.

The solution was loaded into a glass syringe which was fitted to a steel needle with a tip diameter of $0.5 \mathrm{~mm}$ whose tip was filed flat. The whole process is in this condition: an anodic voltage of $19 \mathrm{kV}$, $25 \mathrm{~cm}$ from the tip of the needle to the collecting equipment, the feed rate of $2 \mathrm{ml} \cdot \mathrm{h}^{-1}$ for precursor solution. The nanofiber was collected on the collector which was covered by a piece of aluminum foil pretreated by dilute $\mathrm{HNO}_{3}(1+9)$ and rinsed with distilled water.

After PS nanofibers were gained, it would be firstly cleaned with ethanol and water. PEDOT fibers are in situ synthesized on the basis of PS fibers which are provided by Suzhou Dongqi Biological Technology. Lastly, the PEDOT nanofibers should be dried after being washed with $5 \mathrm{mg}$ ethanol and distilled water. The morphologies of PS nanofibers and PEDOT nanofibers were show as Fig.1.
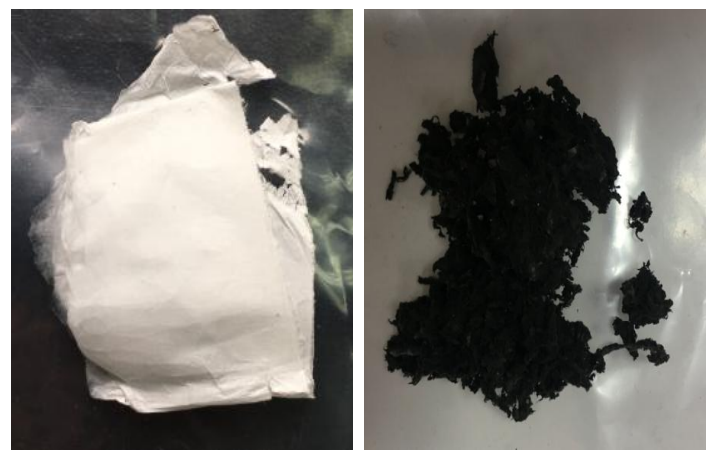

Fig.1 The morphology of PS and PEDOT nanofibers

\section{Preparation of SPE column}

The solid phase extraction (SPE) device was designed for pretreatment of the aqueous sample. As shown in Fig.2, $10 \mathrm{mg}$ of nanofiber were packed into a column with an inside diameter of $2 \mathrm{~mm}$. A liquid storage cartridge was attached to the column. The pressurizer was vailable by using a syringe with a modified tip which was fitted to the liquid storage cartridge. 


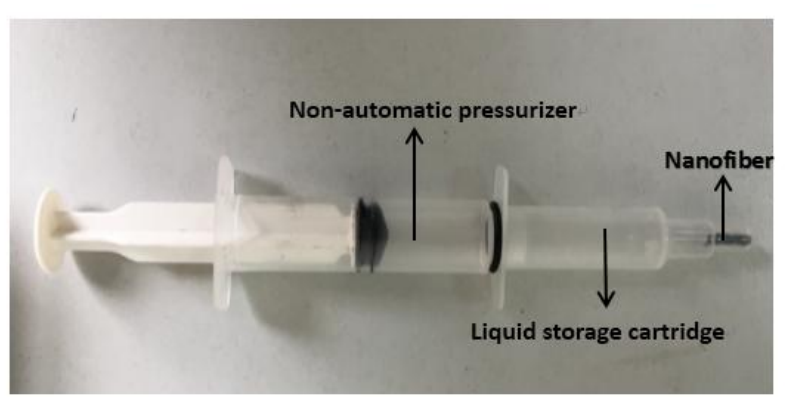

Fig.2 The solid phase extraction device

\section{Procedures}

The nanofiber should be activated before being used. Before activation, the pretreatment device consisted of the column and the liquid storage cartridge was washed with $0.1 \mathrm{~mol} \cdot \mathrm{L}^{-1}$ nitric acid and water orderly, so as to clean up the metal ion remaining in the device. $0.2 \mathrm{~mL} 2.0 \mathrm{~mol} \cdot \mathrm{L}^{-1}$ sodium hydroxide aqueous solution was used to activate the nanofiber and $0.2 \mathrm{~mL}$ deionized water followed. After activation, the column was loaded with $2 \mathrm{~mL}$ of sample solutions. $0.1 \mathrm{~mL}$ of $0.1 \mathrm{~mol} \cdot \mathrm{L}^{-1}$ nitric acid was used as eluent. The flow rate was carefully controlled in a slow dropwise manner in the adsorption and desorption procedures. In order to facilitate the detection and analysis of the instrument, the eluent is diluted with $0.1 \mathrm{~mol} \cdot \mathrm{L}^{-1}$ nitric acid to $1 \mathrm{~mL}$ to be measured.

The concentrations of metal ion were assayed in triplicates by use of ICP-MS with a flow injection system. A series of standards were prepared for instrumental calibration by diluting commercial standards containing $1000 \mu \mathrm{g} \cdot \mathrm{L}^{-1}$ of the metals. Standard and blank samples were determined in the same manner as the samples.

\section{Results and discussion}

\section{Characterization}

The surface characteristics of nanofibers were investigated by the scanning electron microscope and illustrated in Fig. 3. The diameters of the nanofibers were in the range of $200-800 \mathrm{~nm}$, and the nanofiber was dense with network structure. Though PS nanofibers were modified with dithizone (DZ), major morphological change was not observed in the view of the SEM images shown in Fig. $3 \mathrm{~b}$, interestingly, the surface of PEDOT nanofibers is rough and their smoothness is decreased compared with PS nanofibers. There were the burr shape of the materials on the nanofiber surface which could increase the specific surface area. These materials maybe made of PEDOT in situ synthesis with PS as the template. 

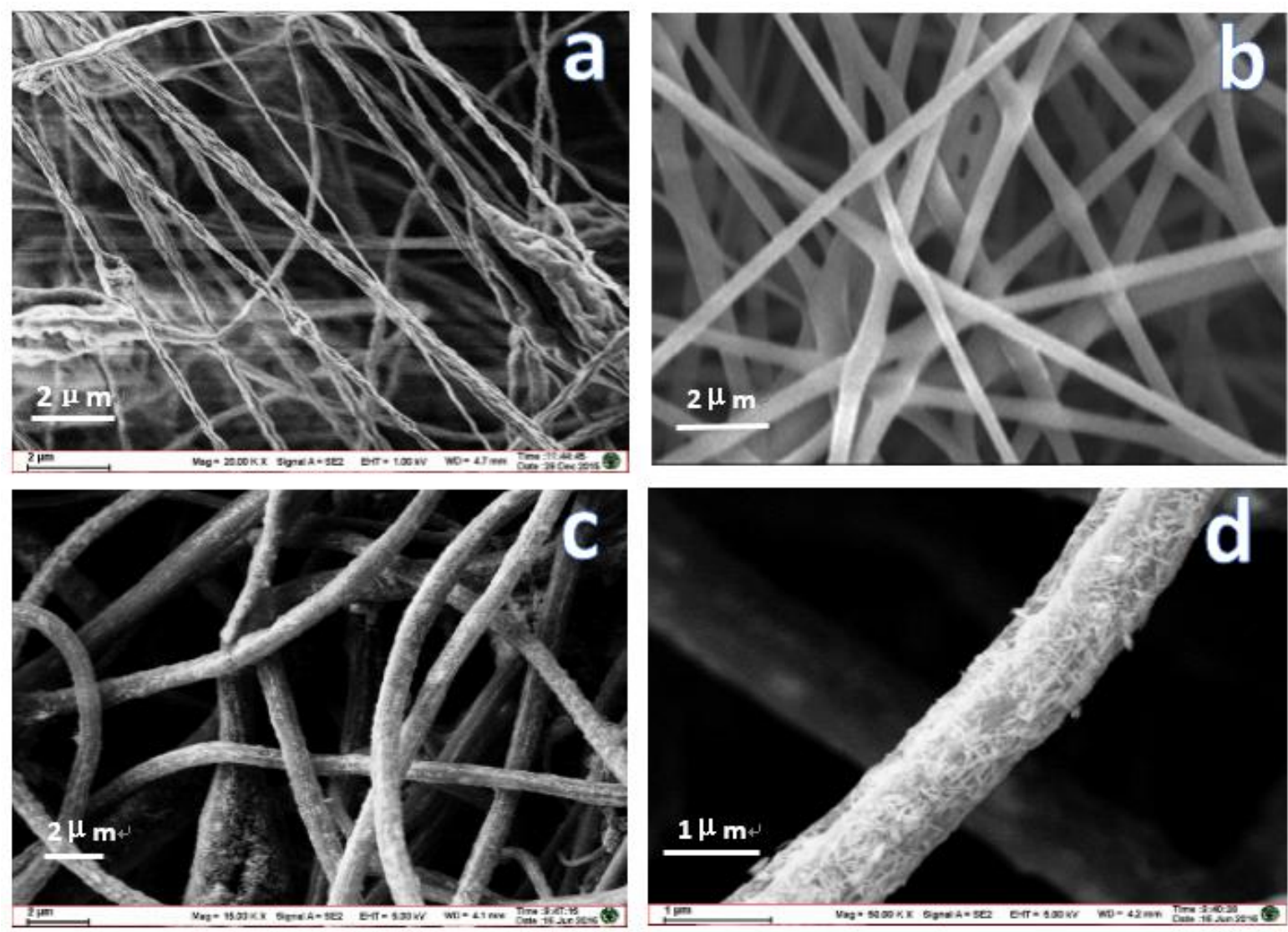

Fig.3 Scanning electron microscope images of nanofibers

(a) PS magnified 20k times, (b) PS-DZ magnified 20k times,

(c) PEDOT (magnified 15k times), (d) PEDOT (magnified 50k times)

\section{Adsorption amount}

The adsorption amount of three kinds of nanomaterials (PS, PS-DZ and PEDOT) for four kinds of heavy metals ( $\mathrm{As}, \mathrm{Pb}, \mathrm{Hg}$ and $\mathrm{Cd}$ ) are shown in Fig.4. PS exhibit markedly lower adsorption amount for all the metal ions. After modified with dithizone reagent, the adsorption amount for the four kinds of metal ions was increased especially for $\mathrm{Cd}$ and As. Among them, PEDOT performs similar adsorption amount with PS-DZ, but PEDOT maybe more prefer to choose as the sorbent because of its better stability and solvent resistance. Therefore, it is indicated that electro-spinning may be a useful method to fabricate selective or broad spectrum nanofibrous adsorbents facilely. The nanofibrous polymer nanomaterial made by electro-spinning of the polymer solution and in situ synthesis was proved to be an effective method for the fabrication of the adsorbent.

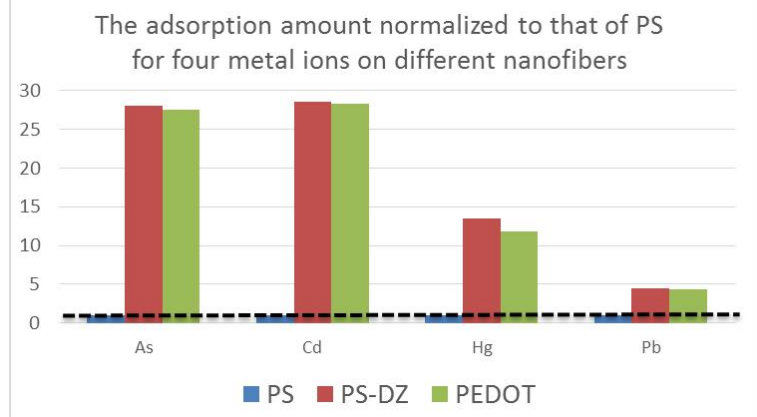

Fig.4 The adsorption amount normalized to that of

PS nanofibers for four metal ions on different nanofibers 


\section{Recovery}

The rate of recovery is an important parameter of the extraction. The recoveries were compared at two concentrations level for four metal ions (1ng $\mathrm{mL}^{-1}$ and $0.1 \mathrm{ng} \mathrm{mL} \mathrm{mL}^{-1}$ ) between PS and PEDOT nanofibers. As shown in Fig.5, it is was known that PEDOT nanofiber appears better recovery rate compared to PS nanofibers, especially for the adsorption at low concentration $\left(0.1 \mathrm{ng} \cdot \mathrm{mL}^{-1}\right)$ level. However, when the concentration of metal ions is increased $\left(1 \mathrm{ng} \cdot \mathrm{mL}^{-1}\right)$, the rate of recovery was not satisfactory for both nanofibers. In other words, It could be determined that PEDOT was suitable for the adsorption of heavy metal ions with low concentration in the actual sample.

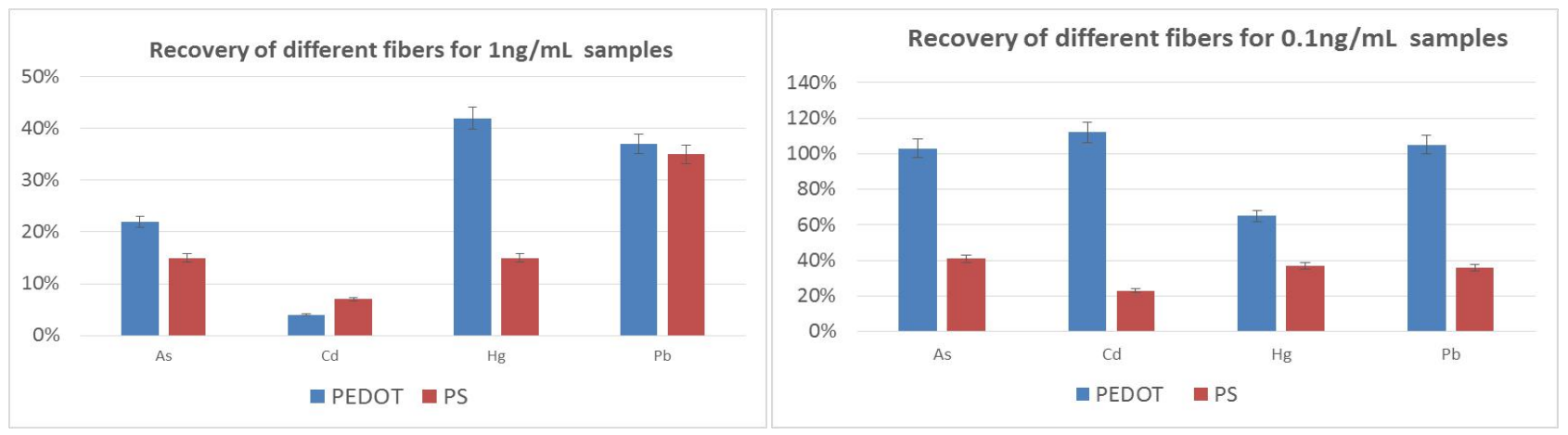

Fig.5 The extraction recovery of different fibers for metal ions at different concentration level

\section{Conclusions}

A new conductive polymer nanofiber was prepared by electrospinning of the polymer solution and in situ synthesis. The adsorption amount and extraction recoveries of three nanofiber for four metal ions were investigated. The enrichment rats of PEDOT for metal ions were satisfactory and higher than that of PS and comparable to PS-DZ nanofibers. What is more, PEDOT was especially suitable for the extraction of heavy metal ions at low concentration level in water. Which is of great significance in analysis and remove of trace heavy metal ions in water. The good environmental stability, easy preparation, and high adsorption ability of conductive polymer nanofiber will contribute to its application in environmental filed.

\section{Acknowledgement}

This study was supported by the National Instrumental Research Program (2014YQ06077303), the National Science Foundation of China (No.81172720, No.81673230); the Social Development Research Program of Jiangsu Province Science and Technology department (Project No. BE2016741); Postgraduate Research \& Practice Innovation Program of Jiangsu Province (No.KYCX17_0189).

\section{References}

[1] C. Duran, A. Gundogdu, V. N. Bulut, M. Soylak, L. Elci, H. B. Senturk, M. Tufekci, Solid-phase extraction of $\mathrm{Mn}(\mathrm{II}), \mathrm{Co}(\mathrm{II}), \mathrm{Ni}(\mathrm{II}), \mathrm{Cu}(\mathrm{II}), \mathrm{Cd}(\mathrm{II})$ and $\mathrm{Pb}$ (II) ions from environmental samples by flame atomic absorption spectrometry (FAAS), J. Hazard.Mater. 146 (2007) 347-355. 
[2] P. K. Roy, A.S. Rawat, V. Choudhary, P. K. Rai, Synthesis and Analytical Application of a Chelating Resin Based on a Crosslinked Styrene/Maleic Acid Copolymer for the Extraction of Trace-Metal Ions, J. Appl. Polym. Sci. 94 (2004) 1771-1779.

[3] Z. P. Zang, Z. Hu, Z. H. Li, Q. He, X. J. Chang, Synthesis, characterization and application of ethylenediamine-modified multiwalled carbon nanotubes for selective solid-phase extraction and preconcentration of metal ions, J.Hazard.Mater. 172 (2009) 958-963

[4] M. Sarkar, M. Das, P. K. Datta, Solid-Phase Extraction for the Decontamination of Alkali Metal, Alkaline Earth Metal, and Ammonium Salts from Heavy Metal Ions, J. Colloid Interf. Sci. 246 (2002) 263

[5] Kang XJ, Pan C, Xu Q, Yao YF, Wang Y, et al. The investigation of electrospun polymer nanofibers as a solid-phase extraction sorbent for the determination of trazodone in human plasma. Anal Chim Acta. 2007; 587: 75-81.

[6] Z. P. Zang, Z. Hu, Z. H. Li, Q. He, X. J. Chang, Synthesis, characterization and application of ethylenediamine-modified multiwalled carbon nanotubes for selective solid-phase extraction and preconcentration of metal ions, J.Hazard.Mater. 172 (2009) 958-963.

[7] Jianjun Deng, Xuejun Kang, LIqin Chen: Journal of Hazardous Materials 196 (2011) 187- 193

[8] I. Al-Saleh, M. Nester, E. DeVol, N. Munchari, S. Health 204 (2001) 165 Chigome S, Torto N. A review of opportunities for electrospun nanofibers in analytical chemistry. Anal Chim Acta. 2011; 706: 25-36. 